\title{
Passivity-Based Control of a Rigid Electrodynamic Tether
}

\section{Larsen, Martin Birkelund; Blanke, Mogens}

Published in:

Journal of Guidance, Control, and Dynamics

Link to article, DOI:

$10.2514 / 1.50446$

Publication date:

2011

Link back to DTU Orbit

Citation (APA):

Larsen, M. B., \& Blanke, M. (2011). Passivity-Based Control of a Rigid Electrodynamic Tether. Journal of Guidance, Control, and Dynamics, 34(1), 118-127. https://doi.org/10.2514/1.50446

\section{General rights}

Copyright and moral rights for the publications made accessible in the public portal are retained by the authors and/or other copyright owners and it is a condition of accessing publications that users recognise and abide by the legal requirements associated with these rights.

- Users may download and print one copy of any publication from the public portal for the purpose of private study or research.

- You may not further distribute the material or use it for any profit-making activity or commercial gain

- You may freely distribute the URL identifying the publication in the public portal

If you believe that this document breaches copyright please contact us providing details, and we will remove access to the work immediately and investigate your claim 


\title{
Passivity-Based Control of a Rigid Electrodynamic Tether
}

\author{
Martin Birkelund Larsen* and Mogens Blanke£ \\ Technical University of Denmark, 2800 Lyngby, Denmark \\ DOI: $\underline{10.2514 / 1.50446}$
}

\begin{abstract}
Electrodynamic tethers provide actuation for performing orbit correction of spacecrafts. When an electrodynamic tether system is orbiting the Earth in an inclined orbit, periodic changes in the magnetic field result in a family of unstable periodic solutions in the attitude motion. This paper shows how these periodic solutions can be stabilized by controlling only the current through the tether. A port-controlled Hamiltonian formulation is employed to describe the tethered satellite system and a passive input-output connection is used in the control design. The control law consists of two parts: a feedback connection, which stabilizes the open-loop equilibrium; and a bias term, which is able to drive the system trajectory away from this equilibrium, a feature necessary to obtain orbit adjustment capabilities of the electrodynamic tether. It is then shown how the periodic solutions of the closed-loop system can be approximated by power series, and a relation is found between control gain and perturbations around the open-loop solution. Stability properties of the system are investigated using Floquet analysis, and the region of stability is found in the plane defined by the control parameters.
\end{abstract}

\section{Nomenclature}

$\boldsymbol{A}=$ system matrix of open-loop system

$\boldsymbol{A}_{k}=$ system matrix related to the feedback loop

$\boldsymbol{A}_{v}=$ system matrix related to the bias term

$\boldsymbol{B}=$ magnetic field of the earth, $\mathrm{T}$

$\boldsymbol{B}_{Z}=$ aligned dipole approximation of $\boldsymbol{B}, \mathrm{T}$

$\boldsymbol{b}=$ input function

$c_{i} \quad=$ short notation for cosine $i$

$\boldsymbol{F}_{e}=$ Lorentz force per unit length tether, $\mathrm{N}$

$g=$ input function in port-controlled Hamiltonian

framework

$H=$ nondimensional Hamiltonian

$\mathcal{H}=$ Hamiltonian, $\mathrm{J}$

$I \quad=$ current through tether, a

$i=$ orbit inclination, rad

$\boldsymbol{J}=$ interconnection matrix

$k=$ controller gain

$k^{*}=$ optimal controller gain

$\mathcal{L}=$ Lagrangian, $\mathrm{J}$

$l=$ length of tether, $\mathrm{m}$

$\boldsymbol{M}=$ monodromy matrix

$m_{A}=$ mass of subsatellite, $\mathrm{kg}$

$m_{B}=$ mass of main satellite, $\mathrm{kg}$

$m_{t} \quad=$ mass of tether, $\mathrm{kg}$

$\boldsymbol{p}=$ nondimensional generalized momenta, $\boldsymbol{p}=\left[\begin{array}{ll}p_{\theta} & p_{\varphi}\end{array}\right]^{T}$

$p_{\theta}=$ generalized momentum associated with $\theta$

$p_{\varphi}=$ generalized momentum associated with $\varphi$

$\boldsymbol{Q}=$ nondimensional generalized force

$\boldsymbol{q}=$ generalized coordinates $\boldsymbol{q}=\left[\begin{array}{ll}\theta & \varphi\end{array}\right]^{T}, \mathrm{rad}$

$\boldsymbol{R}=$ damping matrix

$r=$ unit vector along the tether

$s_{i} \quad=$ short notation for $\sin i$

$u=$ control input

Received 22 April 2010; revision received 5 August 2010; accepted for publication 8 August 2010. Copyright $\odot 2010$ by Martin B. Larsen, Mogens Blanke, and the Technical University of Denmark. Published by the American Institute of Aeronautics and Astronautics, Inc., with permission. Copies of this paper may be made for personal or internal use, on condition that the copier pay the $\$ 10.00$ per-copy fee to the Copyright Clearance Center, Inc., 222 Rosewood Drive, Danvers, MA 01923; include the code and \$10.00 in correspondence with the CCC.

*Ph.D. Graduate, Department of Electrical Engineering, Automation, and Control Group, Elektrovej Building 326; mbl@elektro.dtu.dk.

${ }^{\dagger}$ Professor, Department of Electrical Engineering, Automation, and Control Group, Elektrovej Building 326; Adjunct Professor, Norwegian University of Science and Technology, 7491 Trondheim, Norway; mb@elektro.dtu.dk.

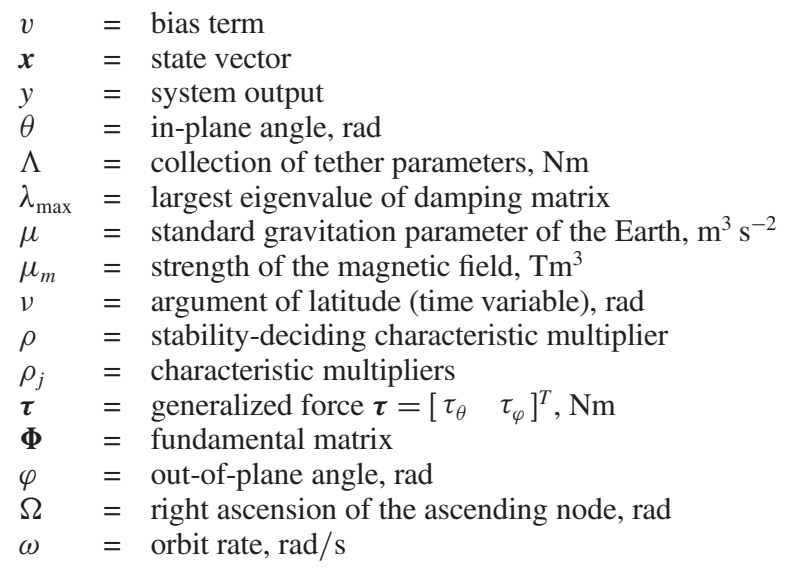

\section{Introduction}

A TETHERED satellite system (TSS) is a system of two or more satellites connected with cables. TSSs have been proposed in connection with numerous different tasks and for several missions, and have therefore been the subject of much research over the last three decades (see [1,2] for reviews). In this work we will consider a TSS consisting of two satellites, a main satellite and a subsatellite, connected by an electrodynamic tether (EDT). An EDT provides a means of performing orbit maneuvers using only electrical power. The system acts as an actuator for the orbit motion by generating an electrodynamic force (the Lorentz force) acting along the tether. By collecting electrons from the surrounding plasma and emitting them from dedicated electron emitters, a current can be led through the tether. Interaction of this current with the magnetic field of the Earth creates a Lorentz force that influences the trajectory of the satellite. This controllable force could be used to perform orbit adjustments, and EDTs have been proposed for deorbiting obsolete satellites [3, 4$]$, for altitude adjustment of the international space station [5] and for reboosting in connection with momentum exchange between satellites [].

There are several control tasks associated with an EDT system. Tether vibration control [7] damps vibration along a flexible tether; orbit control [ $[\underline{8}, \underline{9}]$ changes one or more of the orbit parameters; and attitude control deals with stabilizing libration of the satellite-tether system $[10,11]$. This work treats the attitude problem. Control of the attitude motion is required in order to use the Lorentz force for the desired orbit corrections. The attitude control problem is difficult because control of current alone leaves the attitude dynamics underactuated, and instantaneous forces are always perpendicular to the 
instantaneous $B$-field. This problem is also well known when dealing with attitude control using magnetic coils [12]. Common assumptions when dealing with the attitude control problem are to model the tether as a rigid rod and ignore the orbit changes caused by the Lorentz force. These assumptions will also be made in this study.

An EDT system was investigated in [13] assuming a constant current was passed through the tether. In this case the Lorentz force, acting as a periodic forcing term, was shown to give rise to unstable periodic solutions of the attitude motion. When driven by a constant current, energy is pumped into the attitude motion and active current control is needed to stabilize the motion. Looking into the deorbiting problem, a simple switching control law was presented in [14], where the current was switched on and off depending on the level of a Lyapunov function. Reference [15] showed that stabilization of the open-loop equilibrium of the $\overline{\mathrm{TSS}}$ was possible using feedback linearization. Because of zeros in the input function, the primary control law contained singularities. This problem was handled by switching to a secondary control law in proximity of a singularity. Generation of a Lorentz force and ability to influence the orbit of a TSS is the prime purpose of an EDT. This requires the tether to carry a nonzero current, but will also drive the attitude system away from its open-loop equilibrium. The ability to stabilize the attitude away from the equilibrium is therefore crucial. An commonly explored control strategy for the attitude dynamics is stabilization of open-loop periodic solutions. Two control laws were investigated by [10] with this purpose. The first approach used a feedback of the difference between the present trajectory and a reference trajectory. The second used time-delayed autosynchronization, where the difference between the present trajectory and a one-period-delayed trajectory was used in the feedback loop. Stabilization was obtained using additional actuators. Examining energy considerations, Williams [16] stabilized the periodic solutions by synchronization between the system energy and the energy of a reference trajectory. Considering the combined attitude and orbit control problem using Gauss's planetary equations for modeling the orbit changes, the recent work [11] used numerical predictive control and time-delayed feedback after having discretized the system dynamics.

This paper suggests a solution to the attitude control problem for the TSS using a passivity-based control law, based on a portcontrolled Hamiltonian (PCH) formulation of the dynamics. A control law that adds damping to the open-loop system is shown to make the open-loop equilibrium asymptotically stable. It is discussed how, by adding a bias term, open-loop periodic solutions can be stabilized. The proposed time-varying control law is shown to have the advantage of being static in the sense that no delayed signals or reference trajectories are used. Properties of the closed-loop periodic solutions are investigated and compared with the open-loop solutions. The work presented in this paper is an extension of results presented in $[17,18]$.

The outline of the paper is as follows: Sec. II derives the equations of motion for the system and formulates it as $\overline{\mathrm{PCH}}$ system such that a passive input-output connection is created. Sections III and IV consider the control design for stabilization of the open-loop equilibrium and the open-loop periodic solutions, and bounds are provided for the region of convergence for the time-varying controller. Finally Sec. V offers conclusions.

The notation $\boldsymbol{r}^{T}$ and $\bar{A}^{T}$ will be used to denote the transpose of a vector $\boldsymbol{r}$ or a matrix $\boldsymbol{A}$. The derivative of a scalar or a vector function ( $H$ or $\boldsymbol{x}$ ) with respect to the argument of latitude will be denoted $\dot{H}$ or $\dot{\boldsymbol{x}}$. The Jacobian of a scalar function will be defined as a column vector, hence $\frac{\partial H}{\partial x}$ is a column vector. Identity matrices are denoted $\boldsymbol{I}$, where dimensions should be clear from the context.

\section{Model}

Consider a TSS consisting of a main satellite and a subsatellite connected by an EDT. The satellites have masses $m_{B}$ and $m_{A}$ and are treated as point masses. The tether has mass $m_{t}$ and length $l$. The system will not be considered during the deployment and retrieval phases, hence the length of the tether is assumed constant. For simplicity it is assumed that the mass of the main satellite comprises the main contribution to the total mass $m_{B} \gg m_{A}+m_{t}$, and the center of mass $(\mathrm{CM})$ of the system can therefore be assumed to coincide with that of the main satellite. Only the effect of the EDT on the attitude motion is modeled, and no additional orbit-perturbing forces are included, hence the CM follows a Keplerian orbit. Further, the system is orbiting the Earth in an assumed circular orbit. The orbit plane of the circular orbit can be described by two of the orbit parameters, the right ascension of the ascending node $\Omega$ and the inclination $i$, illustrated in Fig. 1 . The orbit position is described by the argument of latitude in this paper, where the orbit is circular with nonzero inclination. The argument of latitude is denoted $v$, which is measured from the direction of the ascending node. In case the inclination was zero, the true longitude would be used instead. The orbit rate $\omega=\frac{\mathrm{d} v}{\mathrm{~d} t}$ is constant and $v$ is therefore adopted as the independent variable of the model. The attitude motion is described in the orbit frame spanned by the $x, y$, and $z$ axes. The $x$-axis is placed along a vector from the Earth to the CM, the $y$-axis is directed along the orbit velocity, and the $z$-axis is normal to the orbit plane. The orbit frame can be found from an Euler rotation of the inertial frame using the angles $\Omega, i$ and $\nu$ as indicated in Fig. 1 . The inertial frame does not rotate with the Earth and is defined by the $X, Y$ and $Z$ axes. The $X$-axis is in the direction of the vernal point, and the $Z$-axis coincides with the rotational axis of the Earth. The attitude of the TSS is described by a unit vector $\boldsymbol{r}$ from the main satellite to the subsatellite. The system has two stable equilibria due to the gravity gradient. They occur when $\boldsymbol{r}$ coincides with the $x$-axis of the orbit frame. In this paper the system is described around the equilibrium along the negative $x$-axis and this equilibrium is referred to as the open-loop equilibrium, even though there are several. The choice of working point is, however, of no importance due to the symmetry of the gravitational potential. Since the tether is of constant length, the tether motion is restricted to a sphere of radius $l$ in the orbit frame. This sphere is described by an inplane angle $\theta$ and an out-of-plane angle $\varphi$, which are adopted as the generalized coordinates of the system, $\boldsymbol{q}=\left[\begin{array}{ll}\theta & \varphi\end{array}\right]^{T}$. The in-plane and the out-of-plane angles are illustrated in Fig. 2. The in-plane angle is the angle between the negative part of the $x$-axis and the tether projection onto the orbit plane. The out-of-plane angle is the angle between the projection and the actual tether position. Using the generalized coordinates, the vector along the tether can be written as

$$
\boldsymbol{r}=\left[\begin{array}{c}
-\cos \theta \cos \varphi \\
-\sin \theta \cos \varphi \\
-\sin \varphi
\end{array}\right]
$$

\section{A. Equations of Motion}

The Lagrangian of the system has been derived in several previous papers (see e.g. [13]) and can be expressed as

$$
\mathcal{L}(\boldsymbol{q}, \dot{\boldsymbol{q}})=\frac{1}{2} \Lambda\left(\dot{\varphi}^{2}+\cos ^{2} \varphi\left((1+\dot{\theta})^{2}+3 \cos ^{2} \theta\right)\right)
$$

where $\Lambda=\frac{1}{3} \omega^{2} l^{2}\left(3 m_{A}+m_{t}\right)$ is a constant formed by the parameters of the system. Besides the kinetic energy, the Lagrangian includes the gravitational potential, the Coriolis potential and the centrifugal

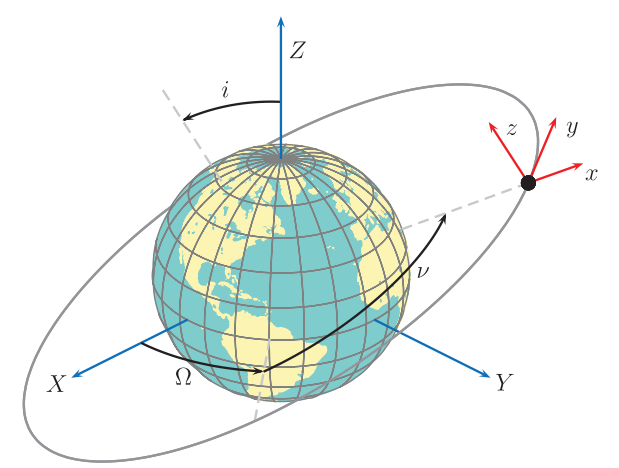

Fig. 1 Orbit description of a circular orbit and the orbit frame. 


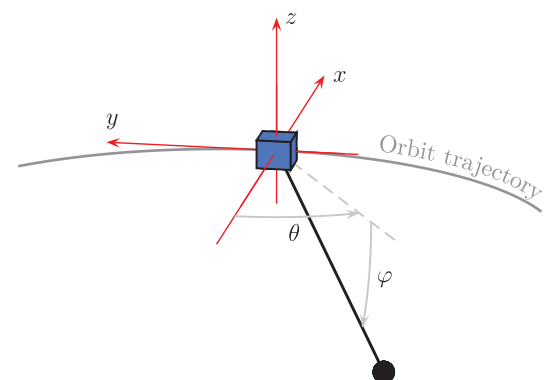

Fig. 2 Definition of the in-plane and the out-of-plane angle in the orbit frame.

potential, represented by the terms $\frac{3}{2} \Lambda \cos ^{2} \theta \cos ^{2} \varphi, \Lambda \dot{\theta} \cos ^{2} \varphi$, and $\frac{1}{2} \Lambda \cos ^{2} \varphi$, respectively. In a Hamiltonian description of the system, the states representing the generalized velocities are replaced by the generalized momenta $\hat{\boldsymbol{p}}=\left[\begin{array}{cc}\hat{p}_{\theta} & \hat{p} \varphi\end{array}\right]^{T}=\frac{\partial \mathcal{L}}{\partial \dot{q}}$. From (2) these are given by

$$
\begin{gathered}
\hat{p}_{\theta}=\Lambda(1+\dot{\theta}) \cos ^{2} \varphi \\
\hat{p}_{\varphi}=\Lambda \dot{\varphi}
\end{gathered}
$$

Using the generalized momenta, the Hamiltonian $\mathcal{H}$ of the system is $\mathcal{H}(\boldsymbol{q}, \hat{\boldsymbol{p}})=\dot{\boldsymbol{q}}^{T} \hat{\boldsymbol{p}}-\mathcal{L}(\boldsymbol{q}, \dot{\boldsymbol{q}}(\boldsymbol{q}, \hat{\boldsymbol{p}}))$,

$$
\mathcal{H}(\boldsymbol{q}, \hat{\boldsymbol{p}})=\frac{1}{2 \Lambda}\left(\hat{p}_{\varphi}^{2}+\frac{\hat{p}_{\theta}^{2}}{\cos ^{2} \varphi}-2 \Lambda \hat{p}_{\theta}-3 \Lambda^{2} \cos ^{2} \theta \cos ^{2} \varphi\right)
$$

To simplify the Hamiltonian, it is scaled by $\Lambda^{-1}$. Furthermore, the generalized momenta are scaled and shifted such that

$$
\begin{gathered}
p_{\theta}=\frac{\hat{p}_{\theta}}{\Lambda}-1 \\
p_{\varphi}=\frac{\hat{p}_{\varphi}}{\Lambda}
\end{gathered}
$$

The shift of $\hat{p}_{\theta}$ is introduced to place the open-loop equilibrium at the origin of the state space. Using these coordinates, the equations of motion are then expressed using Hamilton's equation:

$$
\begin{gathered}
\dot{\boldsymbol{q}}=\frac{\partial H}{\partial \boldsymbol{p}} \\
\dot{\boldsymbol{p}}=-\frac{\partial H}{\partial \boldsymbol{q}}+\boldsymbol{Q}
\end{gathered}
$$

where $\boldsymbol{p}$ is introduced as $\boldsymbol{p}=\left[\begin{array}{ll}p_{\theta} & p_{\varphi}\end{array}\right]^{T}$ and $\boldsymbol{Q}=\frac{\boldsymbol{\tau}}{\Lambda}$ is a nondimensional version of the generalized force. The Hamiltonian is dimensionless and independent of the system parameters:

$$
H(\boldsymbol{q}, \boldsymbol{p})=\frac{1}{2}\left(p_{\varphi}^{2}+\frac{\left(p_{\theta}+1\right)^{2}}{\cos ^{2} \varphi}-2 p_{\theta}-3 \cos ^{2} \theta \cos ^{2} \varphi\right)+1
$$

Here, a constant +1 is added, without loss of generality, to create a positive definite Hamiltonian around the origin. Inserting in (6) the equations of motion are

$$
\begin{gathered}
\dot{\theta}=\frac{p_{\theta}+1}{\cos ^{2} \varphi}-1 \\
\dot{\varphi}=p_{\varphi} \\
\dot{p}_{\theta}=-\frac{3}{2} \cos ^{2} \varphi \sin 2 \theta+Q_{\theta} \\
\dot{p}_{\varphi}=\frac{p_{\theta}+1}{\cos ^{2} \varphi} \tan \varphi-\frac{3}{2} \cos ^{2} \theta \sin 2 \varphi+Q_{\varphi}
\end{gathered}
$$

The unforced system $(\boldsymbol{Q}=\mathbf{0})$ has four equilibria, which can be determined from the critical points of the Hamiltonian. All equilibria are situated in the orbit plane $\left(\varphi^{*}=0\right)$ and have zero momenta $\boldsymbol{p}^{*}=\mathbf{0}$. The system has two stable equilibria placed at $\theta^{*}=0$ and $\theta^{*}=\pi$. The remaining two $\theta^{*}= \pm \frac{\pi}{2}$ are saddle nodes and are unstable.

\section{B. Actuation}

The system is actuated by a current through the tether. The current interacts with the magnetic field of Earth and gives rise to a mechanical force acting along the tether. An essential part of the actuator model is the modeling of the $B$-field of the Earth. The $B$-field can be modeled using a spherical harmonic expansion (see $[19,20]$ ). A dipole model is a spherical harmonic expansion of degree 1 and is commonly employed for analytical derivations to avoid the complexity of a higher order spherical harmonic expansion. The dipole model can be written as the sum of three components, each of which are dipoles directed along the axes in an Earth-fix coordinate system:

$$
\boldsymbol{B}=\boldsymbol{B}_{X}+\boldsymbol{B}_{Y}+\boldsymbol{B}_{Z}
$$

The main contribution to the $B$-field comes from the dipole $\boldsymbol{B}_{Z}$ aligned with the rotational axis of the Earth. The remaining dipoles cause $\boldsymbol{B}$ to be tilted from the rotational axis of the Earth. This reflects the fact that the magnetic north pole of the Earth does not coincide with the geographical north pole. An upper limit for the magnitude of perturbations caused by $\boldsymbol{B}_{X}$ and $\boldsymbol{B}_{Y}$ was provided in [21]. It is common to use $\boldsymbol{B}_{Z}$ to model the $B$-field, because this choice leads to a model independent of the rotation of the Earth. This study also adopts $\boldsymbol{B}_{Z}$ as the $B$-field model, but $\boldsymbol{B}$ will be used in simulations to investigate the influence of perturbations to the $B$-field. By evaluating the dipole model at the origin of the orbit frame, the dipole $\boldsymbol{B}_{Z}$ is expressed in the orbit frame:

$$
\boldsymbol{B}_{Z}=\frac{\mu_{m}}{R^{3}}\left[\begin{array}{c}
-2 \sin v \sin i \\
\cos v \sin i \\
\cos i
\end{array}\right]
$$

where $\mu_{m}$ is the strength of the dipole and $R$ is the radius of the orbit. The electrodynamic force acting on the tether is the Lorentz force, which is proportional to the cross product between a tangent vector to the tether and the $B$-field vector. Since the tether is assumed to be rigid, the position vector $\boldsymbol{r}$ forms a tangent vector at every point along the tether and the Lorentz force per unit length tether becomes

$$
\boldsymbol{F}_{e}=I \boldsymbol{r} \times \boldsymbol{B}
$$

where $I$ is the current through the tether. In the sequel, it is assumed that $I$ can be controlled without limitations and the $B$-field is constant over the length of the tether. To find the generalized force $\tau=$ $\left[\begin{array}{ll}\tau_{\theta} & \tau_{\varphi}\end{array}\right]^{T}$ caused by the Lorentz force, Eq. (11) is projected onto the generalized coordinates. Inclusion of the contribution from the entire tether requires integration along the tether:

$$
\begin{aligned}
\tau_{\theta} & =\int_{0}^{l} \boldsymbol{F}_{e}^{T} \frac{\partial(s \boldsymbol{r})}{\partial \theta} \mathrm{d} s \\
\tau_{\varphi} & =\int_{0}^{l} \boldsymbol{F}_{e}^{T} \frac{\partial(s \boldsymbol{r})}{\partial \varphi} \mathrm{d} s
\end{aligned}
$$

Using (12), the nondimensional generalized force has the affine form:

$$
\boldsymbol{Q}=\boldsymbol{b}(v, \boldsymbol{q}) u
$$

The input $u$ is a dimensionless quantity proportional to the current through the tether:

$$
u=\frac{3}{2} \frac{1}{3 m_{A}+m_{t}} \frac{\mu_{m}}{\mu} I
$$


where $\mu$ is the standard gravitation parameter of the Earth. Considering $\boldsymbol{b}(\boldsymbol{q}, v)=\left[\begin{array}{ll}b_{\theta} & b_{\varphi}\end{array}\right]^{T}$ as input function to the system,

$$
\begin{aligned}
& b_{\theta}(\nu, \boldsymbol{q})=\cos \varphi \sin \varphi \sin i(\cos \nu \sin \theta-2 \sin \nu \cos \theta) \\
& -\cos ^{2} \varphi \cos i \\
& \quad b_{\varphi}(\nu, \boldsymbol{q})=\sin i(\cos \theta \cos \nu+2 \sin \theta \sin \nu)
\end{aligned}
$$

The zeros of the input function are important for the actuation and control design of the system. The input function was analyzed and conditions for zeros were stated in [17].

The model is under-actuated because it has two degrees of freedom and only one control input. Consequently, the magnitude of the Lorentz force can be controlled by the current, but the direction is determined by the states and the direction of the magnetic field. The system description is time-periodic, because the TSS is orbiting through the static magnetic field. Using a more advanced model of the magnetic field, the system description would still be timeperiodic, but the period would be a combination of the orbit period and the period of the rotation of the Earth. It is observed that the Hamiltonian of the system is time-invariant when the orbit is circular.

\section{Passive Input-Output Description}

The control design presented in this paper is based on a passive input-output connection, which is the subject of this section. The equations of motion (6) can be written in a compact form as

$$
\dot{\boldsymbol{x}}=\boldsymbol{J} \frac{\partial H}{\partial \boldsymbol{x}}+\boldsymbol{g}(v, \boldsymbol{q}) u
$$

where $\boldsymbol{x}=\left[\begin{array}{ll}\boldsymbol{q}^{T} & \boldsymbol{p}^{T}\end{array}\right]^{T}$ is the state vector and $\boldsymbol{g}(v, \boldsymbol{q})=$ $\left[\begin{array}{lll}\mathbf{0}^{T} & \boldsymbol{b}^{T} & (\nu, \boldsymbol{q})\end{array}\right]^{T}$. The square matrix $\boldsymbol{J}$ is the symplectic identity:

$$
\boldsymbol{J}=\left[\begin{array}{cc}
0 & \boldsymbol{I} \\
-\boldsymbol{I} & 0
\end{array}\right]
$$

In a general context, the formulation can be expanded to include damping and an output $y$ :

$$
\begin{gathered}
\dot{\boldsymbol{x}}=(\boldsymbol{J}-\boldsymbol{R}) \frac{\partial H}{\partial \boldsymbol{x}}+\boldsymbol{g}(v, \boldsymbol{q}) u \\
y=\boldsymbol{g}^{T}(v, \boldsymbol{q}) \frac{\partial H}{\partial \boldsymbol{x}}
\end{gathered}
$$

The matrix $\boldsymbol{J}=-\boldsymbol{J}^{T}$ is called the interconnection matrix and $\boldsymbol{R}=$ $\boldsymbol{R}^{T} \geq 0$ is the damping matrix. Both matrices can be functions of state variables and time. The matrix $\boldsymbol{J}$ describes how the system is interconnected which in case of a mechanical system like the EDTs is given as (17), and $\boldsymbol{R}$ describes the damping in the system. In the present model damping, e.g., air drag is not taken into account, but the matrix will occur later to describe the damping added in the closed-loop system. This system formulation is called a PCH (see [22]) and a salient feature is that the input-output connection is passive. The input $u$ and the output $y$ is called the port power variables. The product $y u$ describe instantaneous power flow of the system due to the control action. This can be seen from the time derivative of the Hamiltonian which are

$$
\dot{H}=-\left(\frac{\partial H}{\partial \boldsymbol{x}}\right)^{T} \boldsymbol{R} \frac{\partial H}{\partial \boldsymbol{x}}+u y
$$

The first term describe the power flow due to the damping. From (19) it is also seen that $u$ and $y$ forms a passive input-output connection, if the Hamiltonian has a lower bound. This section has presented the system for a single input, but it is similar for the multi-input case.

\section{Stabilization of Open-Loop Equilibrium}

Having formulated the system with a passive input-output relation, the stabilization of the origin can be obtained under conditions described next, by the simple feedback law (see [22] Corollary 3.3 .1$, p. 44$)$ :

$$
u=-k y, \quad k>0
$$

This can be seen by using the Hamiltonian $H$ as a Lyapunov function. The quantity $H$ has been defined as a positive definite function around the open-loop equilibrium and its derivative with respect to $v$ is

$$
\dot{H}=\left(\frac{\partial H}{\partial \boldsymbol{x}}\right)^{T} \boldsymbol{J} \frac{\partial H}{\partial \boldsymbol{x}}+\left(\frac{\partial H}{\partial \boldsymbol{x}}\right)^{T} \boldsymbol{g}(v, \boldsymbol{q}) u=-k y^{2}
$$

Hence, the derivative of $H$ is negative semidefinite. The first term vanishes due to the structure of the interconnection matrix. If the system is zero-state observable, the derivative will be negative definite and asymptotic stability is guaranteed. The zero-state observability condition can be relaxed to zero-state detectability, following [22]. Zero-state observability can be formulated as: A lasting zero in the output $(y=0)$ of the unforced dynamics $(u=0)$ implies that the system is at the zero-state $x=0$. Because of the definition of $y$, the zeros of the input function (15) are essential to fulfill the zero-state observability condition. The zeros induced by the time-varying input have no influence on the zero-state observability because these are countable. The zeros induced by the generalized coordinates have no influence either, which is best seen by writing the output as

$$
y=\boldsymbol{b}^{T}(v, \boldsymbol{q}) \dot{\boldsymbol{q}}=b_{\theta}(v, \boldsymbol{q}) \dot{\theta}+b_{\varphi}(v, \boldsymbol{q}) \dot{\varphi}
$$

If $\boldsymbol{q}$ induces a zero in the input function when $\dot{\boldsymbol{q}} \neq 0$, the open-loop dynamics will lead the system trajectory away from this point, because the origin is the only equilibrium in the region of interest. This also shows that $\dot{\boldsymbol{q}}=0$ cannot induce a lasting zero, except for the open-loop equilibria. The inclination $i$ can also cause a zero in $b_{\theta}$ and $b_{\varphi}$ for equatorial $\left(i=0^{\circ}\right)$ or polar orbits $\left(i=90^{\circ}\right)$, respectively. These situations are hard to handle because either the in-plane or the out-of-plane dynamics are unactuated. However, these conditions are not of great practical importance because they occur as an effect of simplifying the model of the magnetic field. Using the control law (20), the closed-loop dynamics can be written as a Hamiltonian system with additional damping using the open-loop Hamiltonian $H$ :

$$
\dot{\boldsymbol{x}}=(\boldsymbol{J}-k \boldsymbol{R}) \frac{\partial H}{\partial \boldsymbol{x}}
$$

Here, the positive semidefinite damping matrix can be written as

$$
\boldsymbol{R}=\boldsymbol{g}(v, \boldsymbol{x}) \boldsymbol{g}^{T}(v, \boldsymbol{x})=\left[\begin{array}{cc}
\mathbf{0} & \mathbf{0} \\
\mathbf{0} & \boldsymbol{D}
\end{array}\right]
$$

The matrix $\boldsymbol{D}=\boldsymbol{b}(v, \boldsymbol{q}) \boldsymbol{b}^{T}(\nu, \boldsymbol{q})$ describes damping added by the controller to the system. The matrix $\boldsymbol{D}$ has one positive eigenvalue $\lambda_{\max }$ and one equal to zero, except in the case where $\boldsymbol{b}=\mathbf{0}$. The lack of full of rank of $\boldsymbol{D}$ is a consequence of the system being underactuated. The energy flow of the closed-loop system becomes

$$
\begin{aligned}
\dot{H} & =\left(\frac{\partial H}{\partial \boldsymbol{x}}\right)^{T}(\boldsymbol{J}-k \boldsymbol{R}) \frac{\partial H}{\partial \boldsymbol{x}}=-k\left(\frac{\partial H}{\partial \boldsymbol{p}}\right)^{T} \boldsymbol{D} \frac{\partial H}{\partial \boldsymbol{p}} \\
& =-k \dot{\boldsymbol{q}}^{T} \boldsymbol{D} \dot{\boldsymbol{q}} \geq-k \lambda_{\max }\|\dot{\boldsymbol{q}}\|^{2}
\end{aligned}
$$

The energy flow is nonpositive and has a lower bound determined from the nonzero eigenvalue of $\boldsymbol{D}$.

One of the advantages of the control law in Eq. (20) is that the zeros in the input function do not lead to singularities in the control. This could potentially lead to a large region of attraction (ROA) for the closed-loop system.

\section{A. Closed-Loop Analysis}

The closed-loop description of the system will be time-periodic due to the periodic changes in the input function. To investigate the 
stability and performance of such a system, Floquet analysis can be used if the system is linear. Linearizing the closed-loop system around the origin leads to the description

$$
\dot{\boldsymbol{x}}=\boldsymbol{A}_{1}(\nu) \boldsymbol{x}
$$

where the system matrix $\boldsymbol{A}_{1}(v)$ is a $T=2 \pi$ periodic function. The system matrix can be written as

$$
\boldsymbol{A}_{1}(v)=\boldsymbol{A}-k \boldsymbol{A}_{k}(v)
$$

where $\boldsymbol{A}$ is the open-loop system matrix and $\boldsymbol{A}_{k}(v)$ is the part originating from the feedback loop:

$$
\begin{gathered}
\boldsymbol{A}=\left[\begin{array}{cccc}
0 & 0 & 1 & 0 \\
0 & 0 & 0 & 1 \\
-3 & 0 & 0 & 0 \\
0 & -4 & 0 & 0
\end{array}\right] \\
\boldsymbol{A}_{k}(v)=\left[\begin{array}{llcc}
0 & 0 & 0 & 0 \\
0 & 0 & 0 & 0 \\
0 & 0 & \cos ^{2} i & -\frac{1}{2} \sin (2 i) \cos v \\
0 & 0 & -\frac{1}{2} \sin (2 i) \cos v & \sin ^{2} i \cos ^{2} v
\end{array}\right]
\end{gathered}
$$

According to Floquet theory, a fundamental matrix $\boldsymbol{\Phi ( v )}$ of (26) fulfills

$$
\boldsymbol{\Phi}(v+T)=\boldsymbol{\Phi}(v) \boldsymbol{M}
$$

where the monodromy matrix $\boldsymbol{M}$ can be written as

$$
M=\Phi^{-1}(0) \Phi(T)
$$

The stability of the system can be determined from the eigenvalues $\rho_{i}$ of $\boldsymbol{M}$, which are referred to as the characteristic multipliers. The system (26) is asymptotically stable if all $\rho_{i}$ are placed inside the unit circle. The multiplier $\rho=\rho_{j}$ where $j=\arg \max _{i}\left|\rho_{i}\right|$ is denoted the stability-deciding multiplier. Remembering that the fundamental matrix fulfills the differential equation:

$$
\dot{\boldsymbol{\Phi}}(v)=\boldsymbol{A}_{1}(v) \boldsymbol{\Phi}(v)
$$

it is seen from (31) that the monodromy matrix can be found from an integration of ( $\overline{32}$ ) over one period of time from the initial conditions $\boldsymbol{\Phi}(0)=\boldsymbol{I}$. The multipliers can be expanded in a power series around a value of $k$ where the multipliers are known $[23,24]$. In this case around $k=0$, where the system equals the unforced open-loop system. To do this, the monodromy matrix is expanded in a power series as well. The open-loop system is time-invariant and the multipliers and the monodromy matrix are therefore easily found for $k=0$. The expansions of $\rho_{3}$ and $\rho_{4}$ are difficult to find because they are equal for $k=0$. The geometric multiplicity equals two, hence in this case there are two independent eigenvectors corresponding to the eigenvalues $\rho_{3}=\rho_{4}=1$. The expansion becomes even more complicated because the eigenvalues do not cross at $k=0$ but only share a tangent. This means that the expansions of $\rho_{3}$ and $\rho_{4}$ are equal in a linear approximation. The expansion of the characteristic multipliers becomes

$$
\begin{gathered}
\rho_{1,2}=e^{ \pm 2 \sqrt{3} \pi j}\left(1-\pi c_{i}^{2} k+\frac{\pi}{12} c_{i}^{2}\left(6 \pi c_{i}^{2} \mp j \sqrt{3}\right) k^{2}\right)+\ldots \\
\rho_{3,4}=1-\frac{\pi}{2} s_{i}^{2} k+\frac{s_{i}^{4}}{96}\left(12 \pi^{2} \pm j 5 \pi\right) k^{2}+\ldots
\end{gathered}
$$

where $j$ is the imaginary unit and $c_{i}$ and $s_{i}$ are short notation for $\cos i$ and $\sin i$, respectively. The multipliers $\rho_{1,2}$ and $\rho_{3,4}$ are associated with the in-plane and the out-of-plane motion, respectively, and their magnitude can be written as

$$
\left|\rho_{1,2}\right|=1-c_{i}^{2} \pi k+c_{i}^{4} \frac{\pi^{2}}{2} k^{2}+\ldots
$$

$$
\left|\rho_{3,4}\right|=1-s_{i}^{2} \frac{\pi}{2} k+s_{i}^{4} \frac{\pi^{2}}{8} k^{2}+\ldots
$$

It is seen that the system is asymptotically stable for small $k>0$. One problem of the expansion is that the radius of convergence is limited. Another problem is that it can be hard to find higher order terms due to difficulties of finding the higher order terms of the monodromy matrix.

An alternative way of finding the characteristic multipliers is to use numerical integration to determine the monodromy matrix from (31). Figure 3 shows the characteristic multipliers $\rho_{i}$ found numerically for increasing controller gain $k$ plotted in the complex plane. The corresponding magnitudes are shown in Fig. 4. The solutions placed on the unit circle for $k=0$ correspond to the open-loop solutions where the in-plane and the out-of-plane dynamics have a natural frequency of $\sqrt{3}$ and 2 as indicated by the eigenvalues of $\boldsymbol{A}$ in (28). It is seen that there exists an optimal controller gain $k^{*}$ which minimizes $|\rho|$. The existence of $k^{*}$ is also indicated by (34). For $k>k^{*}$ the stability-deciding multiplier is seen to converges toward the unit circle. It is noted that $\rho$ stays inside the unit circle for all $k>0$, hence the system is asymptotically stable for all $k>0$, which is in agreement with the result found using $H$ as a Lyapunov function.

Figure 5 shows the stability-deciding multiplier as a function of the controller gain for different inclinations. The qualitative behavior of the multiplier shown in Fig. 5 follows the behavior seen in Fig. 4, with the exception of a polar or an equatorial orbit. In these cases the

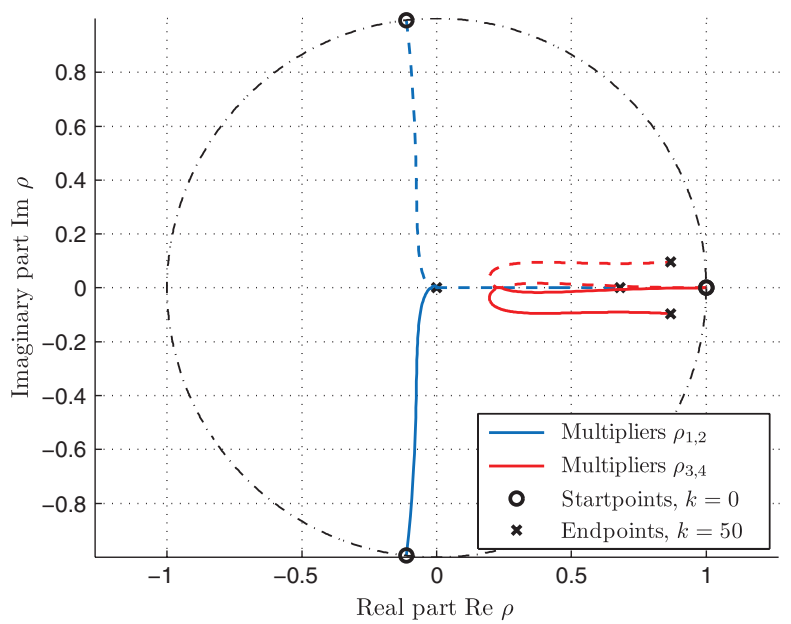

Fig. 3 Characteristic multipliers $\rho_{i}$ as function of $k$ for a fix inclination of $i=45^{\circ}$.

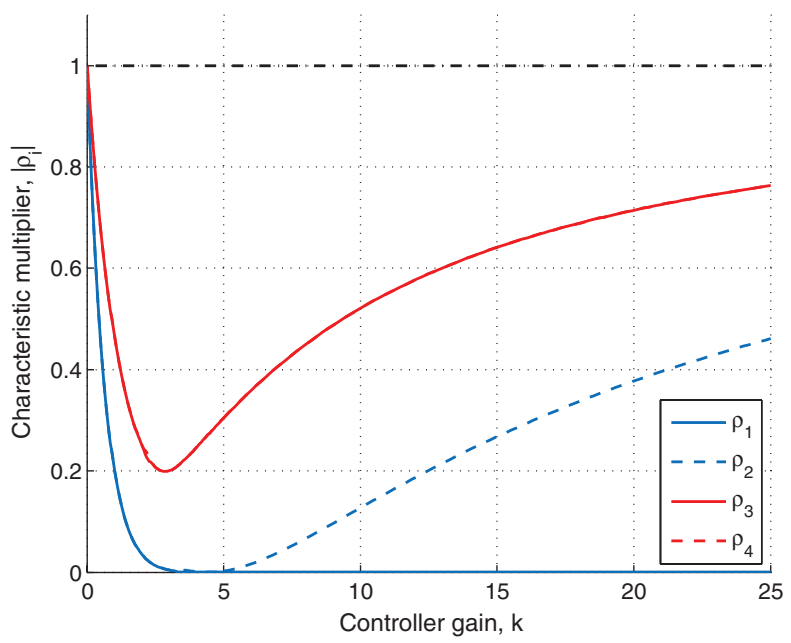

Fig. 4 Absolute value of $\rho_{i}$ as function of $\boldsymbol{k}$ for a fix inclination of $i=45^{\circ}$. 


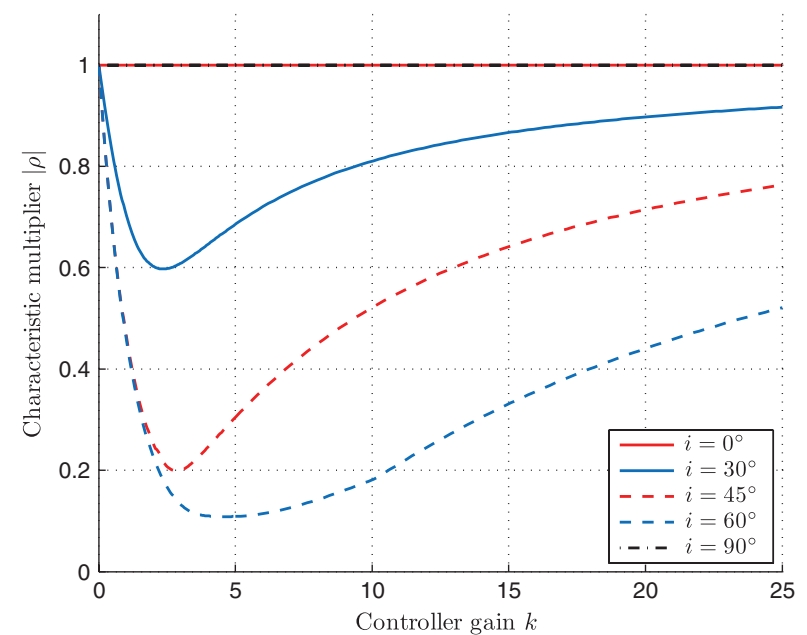

Fig. 5 Absolute value of the stability-deciding multiplier for different inclinations.

stability-deciding multiplier lies on the unit circle for all $k$ as a consequence of the unactuated in-plane and out-of-plane dynamics, respectively. This is also confirmed from the expansion (34) where higher order terms of the in-plane and the out-of-plane multipliers are multiplied by a factor $\cos ^{2} i$ and $\sin ^{2} i$, respectively.

The proposed control law preserves all four open-loop equilibria, so the closed-loop system can only be asymptotically stable, global asymptotical stability can not be obtained. An estimation of the ROA, the range of initial conditions $\boldsymbol{x}_{0}$ for which the system will converge to the origin, is therefore crucial in the evaluation of the control design. In this connection it is important to observe that the control law is the same for the two stable equilibria. It is therefore expected that the state space is divided equally between the regions of attraction around the two stable equilibria.

An obvious way to estimate the ROA is to use the Hamiltonian of the system. If the trajectory gets trapped in the potential well around the origin, the nonincreasing energy level guaranteed by the controller will lead the trajectory to the origin. To be able to escape the potential well, the energy level must be greater than the energy level $H^{*}$ of the separatrices in state space. The energy level can be found from the energy at the saddle nodes that divides the potential wells of the stable equilibria (see Fig. 6). All initial conditions $\left(\boldsymbol{q}_{0}\right.$, $\left.\boldsymbol{p}_{0}\right)$ around the equilibrium with $H\left(\boldsymbol{q}_{0}, \boldsymbol{p}_{0}\right)<H^{*}$ lie in the ROA for the equilibrium. Since the Hamiltonian is independent of the orbit inclination, the estimation of the ROA will be independent of inclination as well. Figure 6 shows the estimate of the ROA in the configuration space for $\dot{\boldsymbol{q}}=\overline{0}$ along with the level curves of $H$.

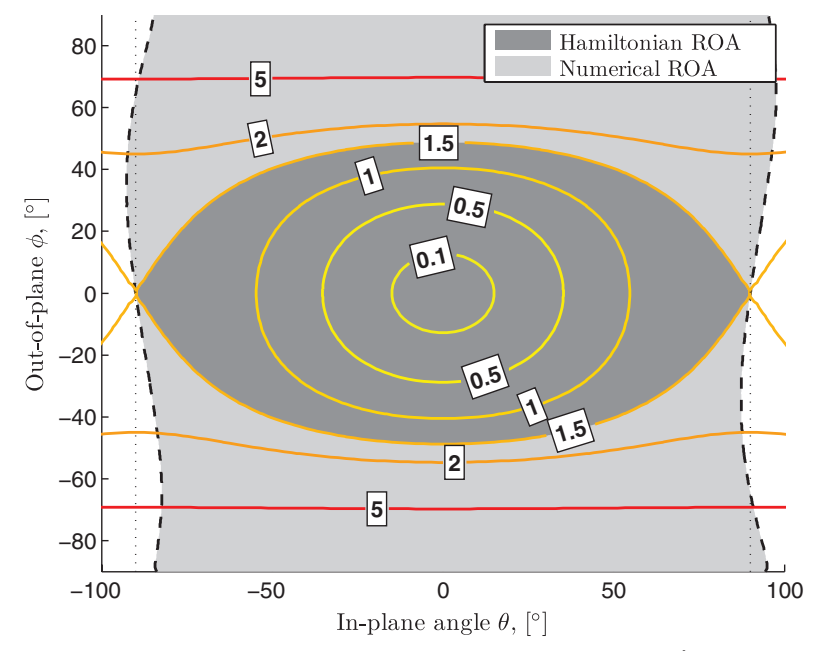

Fig. 6 ROA shown in the configuration space for $\dot{q}=0$.
Figure 6 also shows the ROA found numerically for the initial time $v_{0}=0$. The shape of the numerically determined ROA is similar for other initial times. The ROA more or less equals the lower hemisphere of the configuration space. The configuration space is divided equally between the ROA of the upper and the lower equilibrium, as expected.

Figure 7 shows two simulations of the convergence toward the energy minimum for different initial times. The initial state is set to $\boldsymbol{q}_{0}=\left[\begin{array}{ll}\frac{\pi}{6} & \frac{\pi}{6}\end{array}\right]^{T}$ and $\dot{\boldsymbol{q}}_{0}=0$. The dashed curves in the Figure show simulations using the nonaligned dipole to model the $B$-field, with different initial positions of the perturbation dipoles. It is seen that the perturbation of the $B$-field results in energy levels slightly perturbed compared with the nominal model, but the converges is unaffected by the perturbation.

The control strategy can handle even more complicated models of the magnetic field, provided that sufficient information on the model is available to the controller. The bound on the energy flow shown in Fig. 7 equals $\lambda_{\max }\|\dot{\boldsymbol{q}}\|^{2}$, which is a scaled version of the lower bound given in (25). It is seen that the energy level of the system is only decreasing when the limit on the energy flow is larger than zero.

\section{Stabilization of Periodic Solutions}

Since it is necessary to lead a nonzero current through the tether to generate a Lorentz force on the system, and this current will force the system away from its open-loop equilibrium, it is necessary to find a closed-loop solution that can stabilize the attitude motion with a nonzero tether current. Stabilization about an arbitrary point in the configuration space is not possible due to the under-actuated nature of the system. Only the control signal $u=0$ will lead to equilibria in the equations of motion. Any other control signal will lead to time variations in either the in-plane or the out-of-plane momentum. Since the closed-loop system describes a continuous vector field, the system trajectory cannot converge to points other than the equilibria. An alternative to a stabilization of a point in the configuration space is to stabilize a periodic trajectory. The periodic open-loop solutions found in [13] are obvious candidates for such trajectories. This strategy was investigated in several papers $[10,11,16]$.

To lead the trajectory away from the open-loop equilibrium and stabilize the open-loop periodic solutions, a bias term $v$ is added to the control law (20):

$$
u=-k y+v, \quad k>0
$$

This control law is motivated by the creation of the open-loop periodic solutions when $k=0$. The unstable open-loop periodic solutions were seen to evolve from the marginally stable open-loop equilibrium when the system was perturbed by a constant input. The intention of the control law is then to create similar periodic solutions from the asymptotically stable closed-loop equilibrium. One could expect that the periodic solutions would inherit the stability
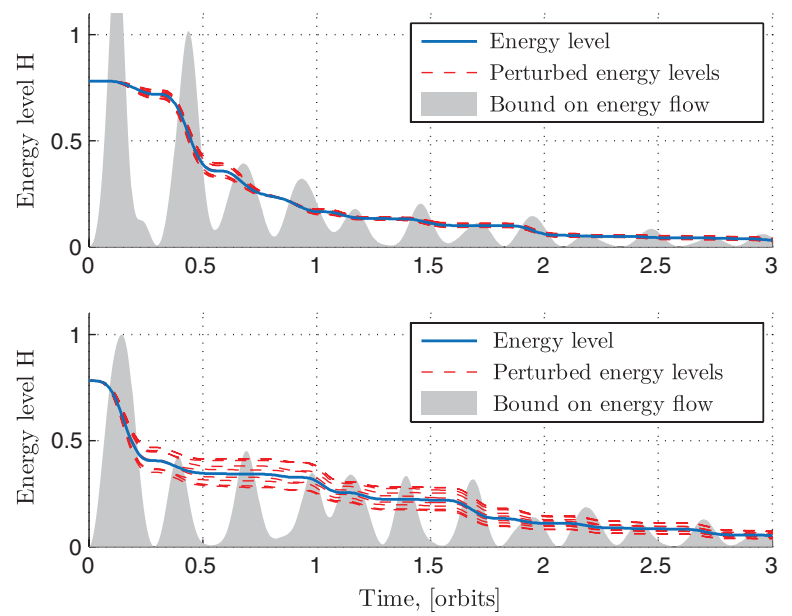

Fig. 7 Simulated energy flow for $v_{0}=0$ and $v_{0}=\frac{\pi}{2}$. 
properties of the closed-loop equilibrium. However, stable periodic solutions only exist for some combinations of the controller gain $k$ and the bias term $v$. The reason is that the two variables will influence the energy flow to the system, and thereby the stability, in different directions.

\section{A. Closed-Loop Analysis}

Using the control law (35), the linear approximation of the closedloop system becomes

$$
\dot{\boldsymbol{x}}=\boldsymbol{A}_{2}(v) \boldsymbol{x}+\boldsymbol{b}_{2}(v) v
$$

where $\boldsymbol{b}_{2}(\nu)$ is a $T=2 \pi$ periodic forcing term originating from the bias:

$$
\boldsymbol{b}_{2}(\nu)=\left[\begin{array}{c}
0 \\
0 \\
-\cos i \\
\sin i \cos v
\end{array}\right]
$$

The system matrix $\boldsymbol{A}_{2}(\nu)$ is also $T=2 \pi$ periodic and can be written as

$$
\boldsymbol{A}_{2}(v)=\boldsymbol{A}-k \boldsymbol{A}_{k}(v)+v \boldsymbol{A}_{v}(v)
$$

where $\boldsymbol{A}$ and $\boldsymbol{A}_{k}(v)$ are given in (28) and (29), respectively, and $\boldsymbol{A}_{v}(\nu)$ is given as

$$
\boldsymbol{A}_{v}(v)=\left[\begin{array}{cccc}
0 & 0 & 0 & 0 \\
0 & 0 & 0 & 0 \\
0 & -2 \sin i \sin v & 0 & 0 \\
2 \sin i \sin v & 0 & 0 & 0
\end{array}\right]
$$

The periodic solutions $\boldsymbol{x}_{p}$ can be approximated by a power series in the parameters $k$ and $v$ using a perturbation method. The solutions for small $k$ and $v$ are approximated around the known solution for $k=v=0$. This is obtained by writing the system matrices and the solution as power series in the parameters. The resulting differential equation is split according to each power and solved, based on the known solution. This approach is a trivial extension of the method presented in [25]. The expansions of the solutions are, to the third order,

$$
\begin{aligned}
\theta(v) & =-\frac{c_{i}}{3} v+\frac{s_{i}^{2}}{3} \sin (2 v) v^{2}+\frac{c_{i} s_{i}^{2}}{6} \sin (2 v) v k \\
+ & \frac{2}{9} c_{i} s_{i}^{2}\left(\cos (2 v)+\frac{1}{3}\right) v^{3}+\frac{s_{i}^{2}}{3}\left(\frac{s_{i}^{2}}{260} \cos (4 v)\right. \\
+ & \left.\frac{7}{3}\left(c_{i}^{2}-\frac{2}{35} s_{i}^{2}\right) \cos (2 v)-\frac{1}{9}\left(c_{i}^{2}+\frac{1}{4} s_{i}^{2}\right)\right) v^{2} k \\
+ & \frac{c_{i} s_{i}^{2}}{13}\left(\frac{s_{i}^{2}}{40} \cos (4 v)+\frac{13}{3}\left(c_{i}^{2}+\frac{1}{30} s_{i}^{2}\right) \cos (2 v)+\frac{13}{216} s_{i}^{2}\right) v k^{2}
\end{aligned}
$$

$$
\begin{aligned}
\phi(v) & =\frac{s_{i}}{3} \cos (v) v-\frac{2 c_{i} s_{i}}{9} \sin (v) v^{2}+\frac{s_{i}^{3}}{36}\left(-\frac{3}{5} \sin (3 v)+\sin (v)\right) v k \\
+ & \frac{s_{i}^{3}}{9}\left(\frac{3}{5} \cos (3 v)+\cos (v)\right) v^{3}+\frac{c_{i} s_{i}^{3}}{9}\left(-\frac{2}{5} \cos (3 v)\right. \\
+ & 2 \cos (v)) v^{2} k-\frac{s_{i}^{3}}{30}\left(\frac{s_{i}^{2}}{56} \cos (5 v)+\left(c_{i}^{2}+\frac{13}{120} s_{i}^{2}\right) \cos (3 v)\right. \\
- & \left.\frac{5}{3}\left(c_{i}^{2}-\frac{1}{20} s_{i}^{2}\right) \cos (v)\right) v k^{2}
\end{aligned}
$$

When no damping is added to the system $(k=0)$, the solutions $(\underline{40})$ coincide with the open-loop periodic solutions found by Peláez et al. [13]. The solutions can be approximated with higher precision by taking the nonlinearities into account. This is done by replacing the nonlinearities with their Taylor expansions and again writing the solution as a power series. The improved solutions are given as

$$
\begin{aligned}
\theta(v) & =-\frac{c_{i}}{3} v+\frac{4 s_{i}^{2}}{9} \sin (2 v) v^{2}+\frac{c_{i} s_{i}^{2}}{6} \sin (2 v) v k \\
+ & \frac{c_{i}}{18}\left(\frac{23}{3} s_{i}^{2} \cos (2 v)+\left(-\frac{4}{9} c_{i}^{2}+s_{i}^{2}\right)\right) v^{3} \\
+ & \frac{s_{i}^{2}}{18}\left(\frac{s_{i}^{2}}{65} \cos (4 v)+20\left(c_{i}^{2}-\frac{7}{150} s_{i}^{2}\right) \cos (2 v)-\frac{1}{3} s_{i}^{2}\right) v^{2} k \\
+ & \frac{c_{i} s_{i}^{2}}{13}\left(\frac{s_{i}^{2}}{40} \cos (4 v)+\frac{13}{3}\left(c_{i}^{2}+\frac{1}{30} s_{i}^{2}\right) \cos (2 v)+\frac{13}{216} s_{i}^{2}\right) v k^{2}
\end{aligned}
$$

$$
\begin{aligned}
\phi(v) & =\frac{s_{i}}{3} \cos (v) v-\frac{2 c_{i} s_{i}}{9} \sin (v) v^{2}+\frac{s_{i}^{3}}{36}\left(-\frac{3}{5} \sin (3 v)+\sin (v)\right) v k \\
+ & \frac{s_{i}}{27}\left(\frac{58}{15} s_{i}^{2} \cos (3 v)+\frac{1}{2}\left(c_{i}^{2}+4 s_{i}^{2}\right) \cos (v)\right) v^{3} \\
+ & \frac{c_{i} s_{i}^{3}}{9}\left(-\frac{3}{5} \cos (3 v)+\frac{5}{3} \cos (v)\right) v^{2} k-\frac{s_{i}^{3}}{30}\left(\frac{s_{i}^{2}}{56} \cos (5 v)\right. \\
+ & \left.\left(c_{i}^{2}+\frac{13}{120} s_{i}^{2}\right) \cos (3 v)-\frac{5}{3}\left(c_{i}^{2}-\frac{1}{20} s_{i}^{2}\right) \cos (v)\right) v k^{2}
\end{aligned}
$$

The solutions (41) agree with the one found in [13] for $k=0$, which illustrates that the closed-loop solutions are perturbed by the controller gain $k$ compared with the open-loop solutions. Note that the in-plane solutions only consist of even multiples of the basic frequency, while the out-of-plane only consists of odd multiples. Consequently, $\theta(v)=\theta(\nu+\pi)$ and $\varphi(v)=-\varphi(v+\pi)$, which geometrically means that the solutions are mirrored in the $\theta$-axis. The solutions are seen to collapse to a point on the $\theta$-axis for an equatorial orbit $\left(i=0^{\circ}\right)$. The approximations of the periodic solutions are found as an eight order power series, which is used when referring to a series approximation.

The solutions (40) and (41) are only valid for small values of $k$ and $v$ due to the convergence properties of the power series. Periodic solutions exist, however, for larger values as well. These solutions can be found by numerical simulations. To investigate the stability of the solutions, the variable $\boldsymbol{\eta}(\nu)=\boldsymbol{x}(\nu)-\boldsymbol{x}_{p}(\nu)$ is introduced to describe a deviation from a periodic solution. Inserting in (36) it is seen that $\eta(v)$ obeys the differential equation:

$$
\dot{\eta}=A_{2}(v) \eta
$$

The equation can be investigated by means of Floquet analysis, which is carried out in the same manner as in the previous section. Using a power series the multipliers become

$$
\begin{aligned}
\rho_{1,2} & =e^{ \pm 2 \sqrt{3} \pi j}\left(1-\pi c_{i}^{2} k+\frac{\pi}{12} c_{i}^{2}\left(6 \pi c_{i}^{2} \mp j \sqrt{3}\right) k^{2}\right. \\
& \left. \pm j s_{i}^{2} c_{i} \frac{\pi \sqrt{3}}{3} k v\right)+\ldots \\
\rho_{3,4} & =1-s_{i}^{2} \frac{\pi}{2} k+\frac{s_{i}^{4}}{96}\left(12 \pi^{2} \pm j 5 \pi\right) k^{2} \\
& \pm j s_{i}^{2} c_{i} \frac{7 \pi}{12} k v \pm j s_{i}^{2} \frac{\pi}{6} v^{2}+\ldots
\end{aligned}
$$

The magnitudes of the multipliers are

$$
\begin{aligned}
& \left|\rho_{1,2}\right|=1-c_{i}^{2} \pi k+c_{i}^{4} \frac{\pi^{2}}{2} k^{2}+\ldots \\
& \left|\rho_{3,4}\right|=1-s_{i}^{2} \frac{\pi}{2} k+s_{i}^{4} \frac{\pi^{2}}{8} k^{2}+\ldots
\end{aligned}
$$

The magnitudes are unchanged compared with (34) by the bias in the second order approximation. This shows that the periodic solutions are asymptotically stable for small $k>0$ and small $v$. The region of stable periodic solutions $(|\rho|<1)$ is shown in the parameter plane 


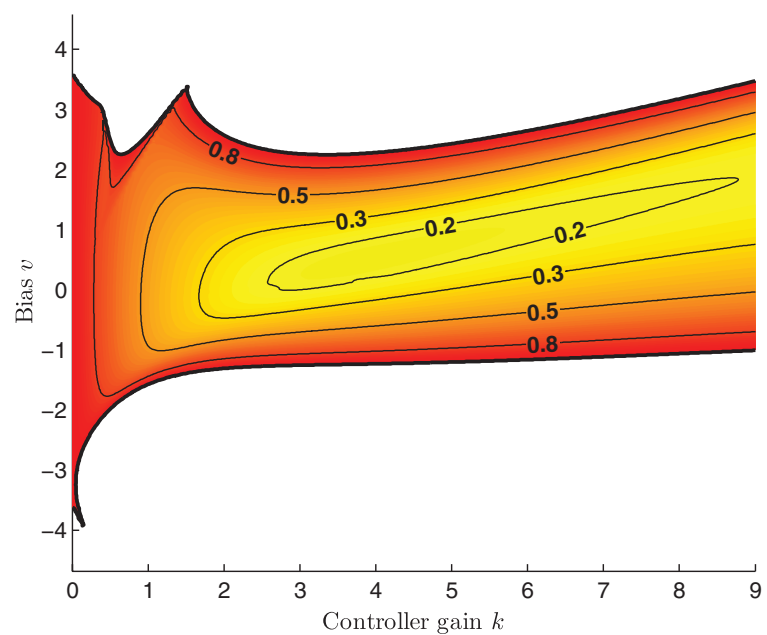

Fig. 8 Stable region in the parameter space for $i=45^{\circ}$.

defined by $k$ and $v$ in Fig. $\underline{8}$. The Figure is based on stability-deciding multipliers found numerically. Stable periodic solutions are seen to exist in a region in the parameter plane and, in agreement with the result from the previous section, the line $v=0$ is included in this region. For each controller gain the bias term has lower and upper limits, which are related to energy flow into the system. However, the relations are quite complex and further scrutiny is not within the scope of this paper.

The approximating series are convenient to show the existence and basic properties of the periodic solutions, as well as to determine initial conditions for simulations. To investigate how well the power series approximates the periodic solution, the error $\varepsilon$ is introduced:

$$
\varepsilon=\frac{1}{2 \pi} \int_{0}^{2 \pi}\left\|\hat{\boldsymbol{x}}(\nu)-\tilde{\boldsymbol{x}}_{p}(\nu)\right\|^{2} \mathrm{~d} \nu
$$

where $\tilde{\boldsymbol{x}}_{p}$ is the series approximation. The solution $\hat{\boldsymbol{x}}$ is found from a nonlinear simulation that has converged to a periodic solution. The error is shown for different parameter values for a third and an eighth order approximation in Figs. 9 and 10. In the case $v=0$, where the solution collapses to the origin, the approximation is exact. The investigation of accuracy of the approximating series requires that the solution is stable and also that the parameters are in the region of converge of the series. Another obstacle is that for $k$ close to zero, convergence of the solution is so slow that computational efforts makes it impractical to determine the magnitude of error. Apart from these limitations, Figs. $\underline{9}$ and 10 show that the approximating series

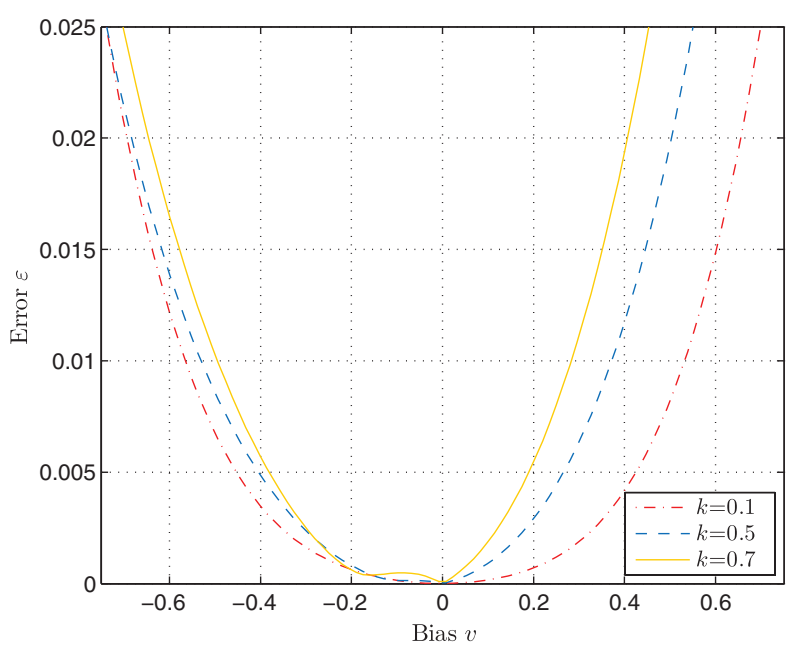

Fig. 9 Error associated with the third order approximation.

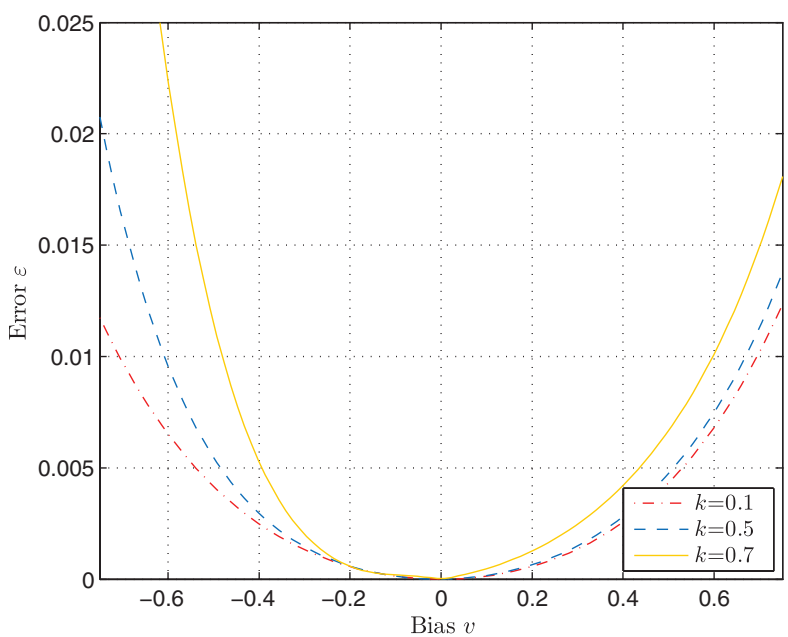

Fig. 10 Error associated with the eighth order approximation.

can be used with reasonable accuracy for parameter ranges shown. It is noted that the limitations associated with the approximating series do not limit the general investigation in this paper as the general results were based on full simulations of the nonlinear system.

Figures 11-14 show simulations of the system for $v=1$, and an inclination of $i=45^{\circ}$. Figure 11 illustrates a simulation of the openloop system $(k=0)$ with an initial value found from the approximated periodic solution. It is seen that this open-loop periodic solution is unstable, even though the instability evolves quite slowly. Figures 12 and 13 shows a simulation of the closed-loop system with $k=\frac{1}{2}$, illustrated in the configuration space and as function of time, respectively. It is seen that the system trajectory converges toward a periodic solution, which resembles the solution approximated by the power series. The stabilized solution is perturbed from the open-loop solution due to the damping injection. Figure 14 shows a simulation of a periodic solution for $k=\frac{1}{2}$ using tilted and nontilted dipole models of the magnetic field of the Earth. Focus on the periodic solution is obtained by removing an initial transient. To avoid a quasi-periodic solution, the simulation was carried out for a satellite with an orbit period of $90 \mathrm{~min}$, hence the periodicity of the system is increased from $2 \pi$ to $32 \pi$, due to the rotation of the Earth. The simulations verify that the controller is able to stabilize a periodic solution with the new period, which is bounded in a region around the unperturbed solution. Since the controller contains no information of a reference solution, the solution is given entirely by the variation in the magnetic field. The mismatch between the $B$-field model used in the controller and the actual one has no significant influence on the stability of the solution.

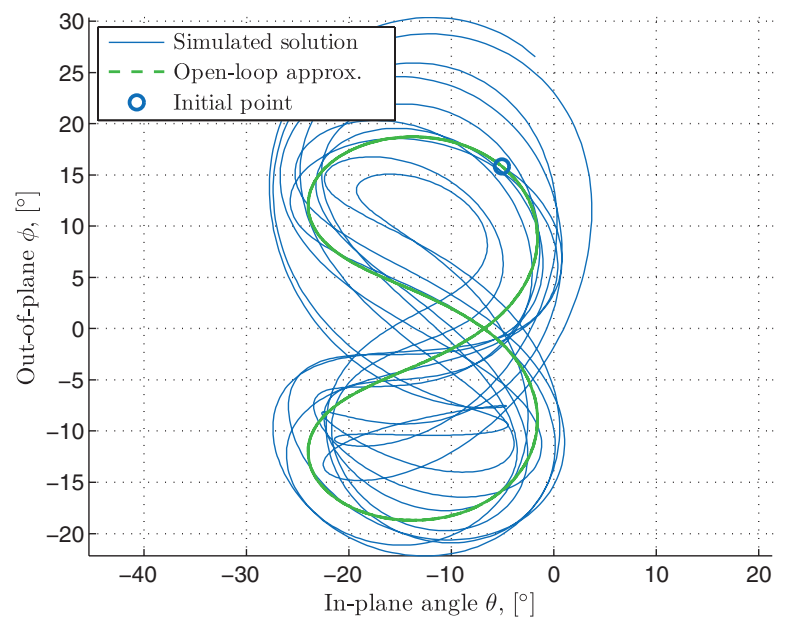

Fig. 11 Open-loop simulation of unstable periodic solution for $k=0$ and $v=1$. 


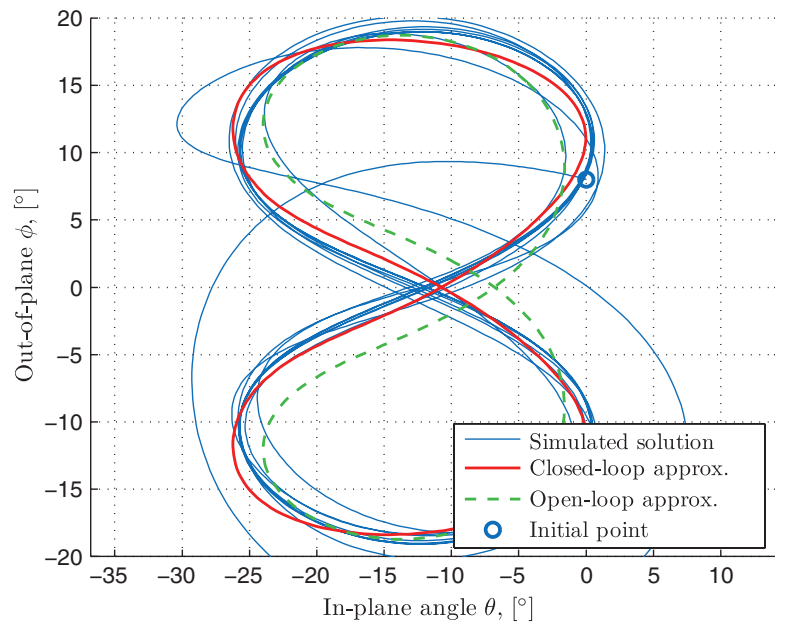

Fig. 12 Converges toward periodic solution for $k=\frac{1}{2}$ in the configuration space.
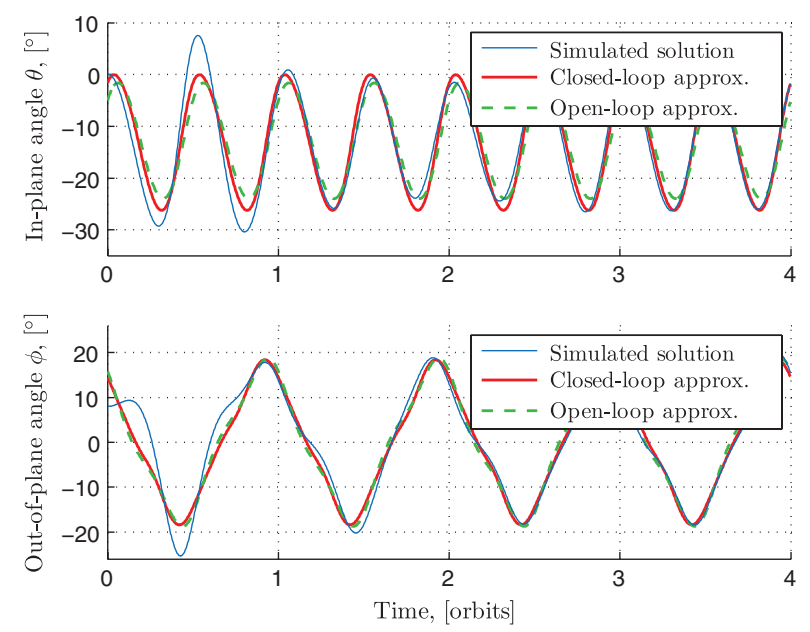

Fig. 13 Converges toward periodic solution for $k=\frac{1}{2}$.

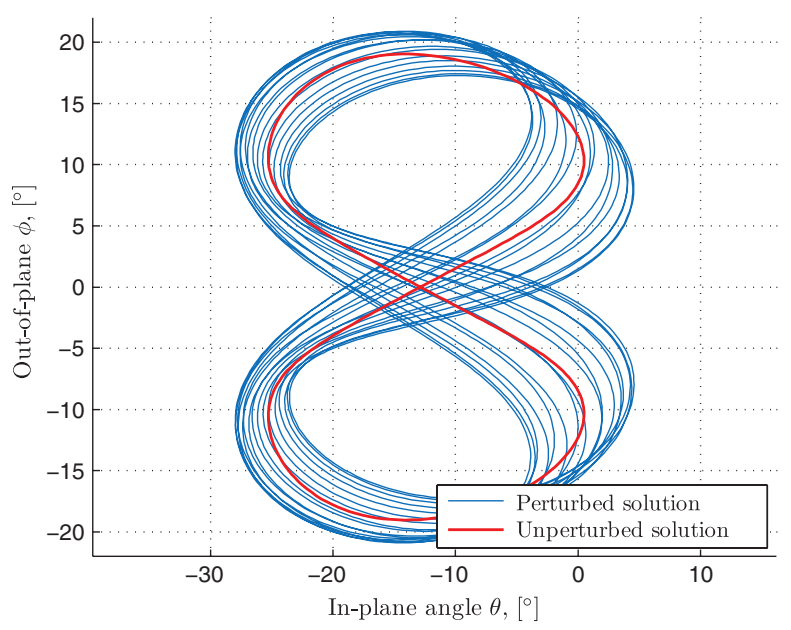

Fig. 14 Periodic solution using different models of the magnetic field.

\section{Conclusions}

This paper developed a control law for stabilizing periodic solutions in the attitude of an EDT system. The time-varying control law was shown to stabilize a family of periodic solutions, which were found to be perturbed versions of a known family of unstable openloop periodic solutions. The control design was based on a passive input-output connection obtained by formulating the equations of motion as a PCH system. The passivity-based formulation allowed zeros in the input function to be handled in a simple manner, without introducing singularities in the control law. The first part of the control law gave stabilization of the open-loop equilibrium. A large ROA was demonstrated for this control law, and it was shown that an optimal control gain exists, providing the fastest convergence toward the equilibrium. An attractive feature of the total control law was shown to be its independence of reference signal and delayed signals. The shapes of the stabilized periodic solutions were investigated using series approximations and numerical simulations. The periodic solutions were found to form symmetric curves around points in the orbit plane, whose distances to the origin were increasing with a bias term in the controller. Stability properties of the controller were investigated by Floquet analysis and the allowable parameter range for stable solutions was determined. The work assumed a simple model of the magnetic field, but the sensitivity to perturbations in the magnetic field was briefly studied. The control law was shown to give an asymptotically stable closed-loop system for all relevant orbits.

\section{References}

[1] Kumar, K. D., "Review of Dynamics and Control of Nonelectrodynamic Tethered Satellite Systems," Journal of Spacecraft and Rockets, Vol. 43, No. 4, 2006, pp. 705-720. doi:10.2514/1.5479

[2] Cartmell, M. P., and McKenzie, D. J., "A Review of Space Tether Research," Progress in Aerospace Sciences, Vol. 44, No. 1, 2008, pp. 121.

doi:10.1016/j.paerosci.2007.08.002

[3] Iess, L., Bruno, C., Ulivieri, C., Ponzi, U., Parisse, M., Laneve, G., Dobrowolny, M., De Venuto, F., Bertotti, B., Anselmo, L., and Vannaroni, G., "Satellite De-Orbiting by Means of Electrodynamic Tethers Part 1: General Concepts and Requirements," Acta Astronautica, Vol. 50, No. 7, 2002, pp. 399-406. doi:10.1016/S0094-5765(01)00180-1

[4] Pardini, C., Hanada, T., Krisko, P. H., Anselmo, L., and Hirayama, H., "Are De-Orbiting Missions Possible Using Electrodynamic Tethers? Task Review from the Space Debris Perspective," Acta Astronautica, Vol. 60, Nos. 10-11, 2007, pp. 916-929.

doi:10.1016/j.actaastro.2006.11.001

[5] Johnson, L., and Herrmann, M., "International Space Station Electrodynamic Tether Reboost Study," NASA, NASA/TM-1998208538, Marshall Space Flight Center, 1998.

[6] Sorensen, K. F., "Conceptual Design and Analysis of an MXER Tether Boost Station," AIAA, Propulsion Research Center/TD40, NASA, Marshall Space Flight Center, 2001.

[7] Pasca, M., "Nonlinear Control of Tethered Satellite System Oscillations," Nonlinear Analysis Theory, Methods and Applications, Vol. 30, No. 6, 1997, pp. 3867-3878. doi:10.1016/S0362-546X(97)00114-4

[8] Tragesser, S. G., and San, H., "Orbital Maneuvering with Electrodynamic Tethers," Journal of Guidance, Control, and Dynamics, Vol. 26, No. 5, 2003, pp. 805-810. doi: $10.2514 / 2.5115$

[9] Williams, P., "Optimal Orbital Transfer with Electrodynamic Tether," Journal of Guidance, Control, and Dynamics, Vol. 28, No. 2, 2005, pp. 369-372. doi:10.2514/1.12016

[10] Peláez, J., and Lorenzini, E. C., "Libration Control of Electrodynamic Tethers in Inclined Orbit," Journal of Guidance, Control, and Dynamics, Vol. 28, No. 2, 2005, pp. 269-279. doi: $10.2514 / 1.6473$

[11] Williams, P., "Libration Control of Electrodynamic Tethers Using Predictive Control with Time-Delayed Feedback," Journal of Guidance, Control, and Dynamics, Vol. 32, No. 4, 2009, pp. 1254 1268 . doi: $10.2514 / 1.41039$

[12] Wisniewski, R., and Blanke, M., "Fully Magnetic Attitude Control for Spacecraft Subject to Gravity Gradient," Automatica, Vol. 35, No. 7, 1999, pp. 1201-1214. doi:10.1016/S0005-1098(99)00021-7

[13] Peláez, J., Lorenzini, E. C., López-Rebollal, O., and Ruiz, M., "A New Kind of Dynamic Instability in Electrodynamic Tethers," Journal of the Astronautical Sciences, Vol. 48, No. 4, 2000, pp. 449-476.

[14] Corsi, J., and Iess, L., "Stability and Control of Electrodynamic Tethers 
for De-Orbiting Applications," Acta Astronautica, Vol. 48, Nos. 5-12, 2001, pp. 491-501.

doi:10.1016/S0094-5765(01)00049-2

[15] Zhou, X., Li, J., Baoyin, H., and Zakirov, V., "Equilibrium Control of Electrodynamic Tethered Satellite Systems in Inclined Orbits," Journal of Guidance, Control, and Dynamics, Vol. 29, No. 6, 2006, pp. 14511454.

doi:10.2514/1.21882

[16] Williams, P., "Energy Rate Feedback for Libration Control of Electrodynamic Tethers," Journal of Guidance, Control, and Dynamics, Vol. 29, No. 1, 2006, pp. 221-223. doi: $10.2514 / 1.17530$

[17] Larsen, M. B., and Blanke, M., "Control by Damping Injection of Electrodynamic Tether System in an Inclined Orbit," Proceedings of the American Control Conference, American Automatic Control Council, St. Louis, MO, 2009, pp. 4824-4829. doi:10.1109/ACC.2009.5159896.

[18] Larsen, M. B., and Blanke, M., "Stabilization of Periodic Solutions in a Tethered Satellite System by Damping Injection," Proceedings of European Control Conference, European Union Control Association, Budapest, 2009, pp. 2169-2174.

[19] Macmillan, S., and Maus, S., "International Geomagnetic Reference Field: The Tenth Generation," Earth, Planets and Space, Vol. 57,
No. 12, 2005, pp. 1135-1140. doi:10.1029/2005EO160006

[20] Wertz, J. R., "Magnetic Field Model," Spacecraft Attitude Determination and Control, 1st ed., Reidel Publishing, 1978, pp. 779-786.

[21] Larsen, M. B., and Blanke, M., "Nonlinear Control of Electrodynamic Tether in Equatorial or Somewhat Inclined Orbits," Proceedings of Mediterranean Conference on Control and Automation, Mediterranean Control Association, Athens, 2007, pp. 1-6. doi:10.1109/ MED.2007.4433876

[22] Schaft, A. v. d., "Hamiltonian Systems as Passive Systems," $L_{2}$-Gain and Passivity in Nonlinear Control, 2nd ed., Springer-Verlag, New York, 2000, pp. 63-123.

[23] Seyranian, A. P., "Sensitivity Analysis of Multiple-Eigenvalues," Mechanics of Structures and Machines, Vol. 21, No. 2, 1993, pp. 261284.

doi:10.1080/08905459308905189

[24] Seyranian, A. P., Solem, F., and Pedersen, P., "Stability Analysis for Multi-Parameter Linear Periodic Systems," Archive of Applied Mechanics, Vol. 69, No. 3, 1999, pp. 160-180. doi: $10.1007 / \mathrm{s} 004190050212$

[25] Grimshaw, R., "Perturbation Methods for Periodic Solutions," Nonlinear Ordinary Differential Equations, 1st ed., Blackwell Science, Boston, 1990, pp. 173-189. 


\section{IMPORTANT: PLEASE READ CAREFULLY.}

\section{Queries}

When production of AIAA journal papers begins, the official approved PDF is considered the authoritative manuscript. Authors are asked to submit source files that match the PDF exactly, to ensure that the final published article is the version that was reviewed and accepted by the associate editor. Once a paper has been accepted, any substantial corrections or changes must be approved by the associate editor before they can be incorporated.

If you and the EIC settled on some final changes to your manuscript after it was accepted, it is possible that your page proofs do not reflect these final changes. If that is the case, please submit these changes as itemized corrections to the proofs.

If final changes were made to the figures, please check the figures appearing in the proofs carefully. While it is usual procedure to use the figures that exist in the source file, if discrepancies are found between figures (manuscript source file vs the approved PDF), the figures from the PDF are inserted in the page proofs, again deferring to the PDF as the authoritative manuscript. If you find that agreed-upon final changes to your figures are not appearing in your page proofs, please let us know immediately.

Q1. Please check that author affiliations and footnotes are correct. For author 1, please clarify "Graduate": is author student or professor?

Q2. Please check that above copyright-statement assignment is correct.

Q3. "Notation" subheading has been deleted. Subheadings are not allowed in Introduction. Please advise if an alternate change is required.

Q4. Lone subheading is prohibited: please advise whether to delete or add second subheading below.

Q5. Lone subheading is prohibited: please advise whether to delete or add second subheading below.

Q6. Please clarify [6] (is this a report? etc.).

Q7. This query was generated by an automatic reference checking system. Reference [13] could not be located in the databases used by the system. While the reference may be correct, we ask that you check it so we can provide as many links to the referenced articles as possible.

Q8. Please provide the city for publisher of [20].. 